\title{
Application of Analytical Hierarchy Process Method in Laptop Selection
}

\author{
Devi Yunita, B. Ayshwarya, Eka Ridhawati, Miftachul Huda, Azmil Hashim, Kamarul Shukri Mat \\ Teh, Phong Thanh Nguyen, K. Shankar, Andino Maseleno
}

\begin{abstract}
The process of selecting a laptop must be based on the capabilities and needs of the buyer. Buyers are faced with a large selection of laptop brands with various specifications, based on that we can make a decision support system aimed to help buyers select a laptop that fits the criteria of Style, Reability and Price, and takes alternatives Samsung, HP and Lenovo. This study used Analytical Hierarchy Process (AHP) method. Obtained results from Samsung, HP (Hewlett-Packard) and Lenovo.
\end{abstract}

Index Terms: Laptop, Decision Support System, Analytical Hierarchy Process

\section{INTRODUCTION}

\section{A. Background}

A laptop is one type of computer that can be carried everywhere, the weight of a laptop depends on its size, the material, and the specifications of the laptop. The components in the laptop are the same as the components on the personal computer (PC) the difference is that the components on the laptop are reduced in size, made lighter, and save power. And as technology advances many laptop brands have sprung up and from every single one launches laptop with their various advantages. Of the various types of laptops, specifications, and functions often cannot be used by consumers who do not meet their needs. Because of this, a laptop selecting system is needed.

The determination process takes account of prices, brands, and laptop specifications such as processor, ram, and memory.

connection with that information relating to the final assignment with the title "Application of Analytical Hierarchy Process Method in Laptop Selection".

\section{B. Formulation of the problem}

Based on the problems found in the background, a problem occur that can be discussed in this final project, namely:

Revised Manuscript Received on July 22, 2019.

Devi Yunita, Department of Information System, STMIK Pringsewu, Lampung Indonesia.

B. Ayshwarya, Department of Computer Science, Kristu Jayanti College, Bangalore-560043, India.

Eka Ridhawati, Department of Information System, STMIK Pringsewu, Lampung Indonesia.

Miftachul Huda, Universiti Pendidikan Sultan Idris, Malaysia.

Azmil Hashim, Universiti Pendidikan Sultan Idris, Malaysia.

Kamarul Shukri Mat Teh, Universiti Sultan Zainal Abidin, Malaysia.

Phong Thanh Nguyen, Department of Project Management, Ho Chi Minh City Open University (HCMCOU), Vietnam.

K. Shankar, School of Computing, Kalasalingam Academy of Research and Education, Krishnankoil, India.

Andino Maseleno, Universiti Tenaga Nasional, Malaysia.
1. How to design a system that supports the decisions of laptop choosing in order to facilitate consumers in choosing a laptop that suits their needs.

\section{Scope of problem}

In order to avoid a discussion outside the problem, then the provisions needed to be able to provide inspiration that is more directed at the problem. The limits of problems are:

1. Creating a laptop selection decision support system.

2. Using the Analytical Hierarchy Process (AHP) method in making this decision support system.

3. Creating this decision support system using the PHP programming language.

\section{Research objective}

From the description of the problem above, the objective of this final project was to create a laptop selecting using decision support system that will help to provide solutions for consumers in choosing a laptop that suits their needs.

\section{E. Benefits of research}

A. Providing the right alternative for choosing a laptop that suits your needs.

b. Extending knowledge about the decision support system with analytical hierarchy method.

\section{METHODOLOGY OF RESEARCH}

Someone will buy a laptop, the alternative laptop that will be chosen is Acer, Toshiba, Apple. While the criteria chosen are Style, Reability, Price. Next do a pairwise comparison with the Saaty Scale to get the criteria weights:

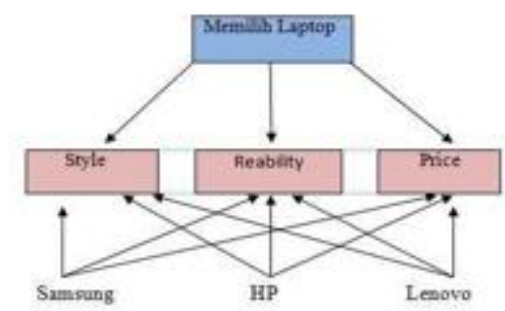

a. Paired comparison with saaty scale

\begin{tabular}{|l|l|l|l|}
\hline & Style & Reability & Price \\
\hline Style & 1 & 3 & 5 \\
Reability & $1 / 3$ & 1 & 2 \\
Price & $1 / 5$ & $1 / 2$ & 1 \\
\hline
\end{tabular}

b. Calculate the weight of the criteria (priority vector) by: 
1) Make the value of the paired comparison be a decimal value and add up each column.

\begin{tabular}{|l|l|l|l|}
\hline & Style & Reability & Price \\
\hline Style & 1.00 & 3.00 & 5.00 \\
Reability & 0.33 & 2.00 & 3.00 \\
Price & 0.20 & 0.50 & 1.00 \\
Collumn & 1.53 & 4.50 & 8.00 \\
& & & \\
\hline
\end{tabular}

Column

$1: 1.00+0.33+0.20=1.53$

2: $3.00+2.00+0.50=4.50$

3: $5.00+3.00+1.00=8.00$

2) normalization of the value of each paired comparison matrix column by dividing each value in the matrix column with the corresponding column sum results.

\begin{tabular}{|l|l|l|l|}
\hline & Style & Reability & Price \\
\hline Style & $\mathbf{0 . 6 5}$ & 0.67 & 0.62 \\
\hline Reability & 0.22 & 0.22 & 0.25 \\
\hline Price & 0.13 & 0.11 & 0.13 \\
\hline
\end{tabular}

How to normalize each value: Column 1 row 1 (1.00) divided by the sum of results per column (1.53) the results enter column 1 row 10.65 and so on until all values are met.

3) Calculate the average value of the sum of each matrix line

\begin{tabular}{|l|l|l|l|l|}
\hline & Style & Reability & Price & $\begin{array}{c}\text { Vector } \\
\text { Scale }\end{array}$ \\
\hline Style & $\mathbf{0 . 6 5}$ & 0.67 & 0.62 & 0.65 \\
Reability & 0.22 & 0.22 & 0.25 & 0.23 \\
Price & 0.13 & 0.11 & 0.13 & 0.12 \\
\hline
\end{tabular}

Obtain vector priority values: Line $1: 0.65+0.67+0.62 / \mathrm{n}=$

0.65 Line $2: 0.22+0.22+0.25 / \mathrm{n}=0.23$ Line $3: 0.13+0.11$

$+0.1113 / \mathrm{n}=0.12$

$\mathrm{n}=$ Number of criteria used.

c. Calculate CI

\begin{tabular}{|l|l|l|l|l|}
\hline & & & & Scale \\
& Style & Reability & Price & Vector \\
\hline Style & 1.00 & 3.00 & 5.00 & $\mathbf{0 . 6 5}$ \\
Reability & 0.33 & 1.00 & 2.00 & $\mathbf{0 . 2 3}$ \\
Price & 0.20 & 0.50 & 1.00 & $\mathbf{0 . 1 2}$ \\
Collumn & $\mathbf{1 . 5 3}$ & $\mathbf{4 . 5 0}$ & $\mathbf{8 . 0 0}$ & \\
\hline
\end{tabular}

$$
]=\operatorname{xam} \lambda 1,53 \times 0,65]+[4,50 \times 0,23]+[8,00 \times 0,12]
$$$$
=3.03
$$

$\mathrm{CI}=(\max -\mathrm{n}) / \mathrm{n}-1$

$=(3,03-3) / 2=0,02$

Table IR

\begin{tabular}{|l|l|l|l|l|l|}
\hline $\begin{array}{l}\text { Ordo } \\
\text { Matrix }\end{array}$ & RI & Ordo & RI & $\begin{array}{l}\text { Ordo } \\
\text { Matrix }\end{array}$ & RI \\
\hline 1 & 0 & 6 & 1.24 & 11 & 1.51 \\
2 & 0 & 7 & 1.32 & 12 & 1.48 \\
3 & 0.58 & 8 & 1.41 & 13 & 1.56 \\
4 & 0.9 & 9 & 1.45 & 14 & 1.57 \\
5 & 1.12 & 10 & 1.49 & 15 & 1.59 \\
\hline
\end{tabular}

So: $\mathrm{CR}=\mathrm{CI} / \mathrm{IR}$

$=0.02 / 0.58$

$=0.03$

d. New hierarchy arrangement (complete with criteria weights)

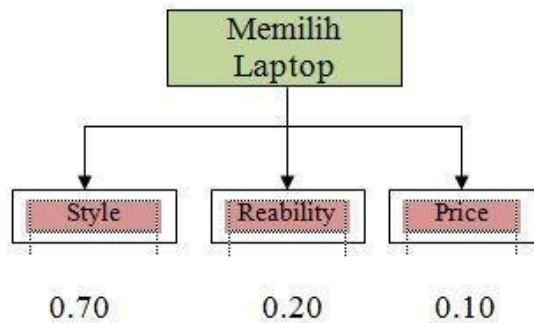

e. Calculation of alternative weights for Style criteria

a. Pairwise comparison

\begin{tabular}{|l|l|l|l|}
\hline Style & Samsung & $\mathrm{Hp}$ & Lenovo \\
Samsung & 1 & 3 & 5 \\
Hp & $1 / 3$ & 1 & $1 / 3$ \\
Lenovo & $1 / 5$ & 3 & 1 \\
\hline
\end{tabular}

b. Criteria weight (vector priority)

Decimal value and sum of each column

\begin{tabular}{|l|l|l|l|}
\hline Style & Samsung & Hp & Lenovo \\
\hline Samsung & 1.00 & 3.00 & 5.00 \\
\hline Hp & 0.33 & 1.00 & 0.33 \\
\hline Lenovo & 0.20 & 3.00 & 1.00 \\
\hline Collumn & 1.53 & 7.00 & 6.33 \\
\hline
\end{tabular}

Normalization

\begin{tabular}{|l|l|l|l|}
\hline Style & Samsung & Hp & Lenovo \\
Samsung & 0.65 & 0.43 & 0.79 \\
Hp & 0.22 & 0.14 & 0.05 \\
Lenovo & 0.13 & 0.43 & 0.13 \\
\hline
\end{tabular}

Get the priority vector

\begin{tabular}{|l|l|l|l|l|}
\hline Style & Samsung & Hp & Lenovo & Priority vector \\
\hline Samsung & 1.00 & 3.00 & 5.00 & 0.62 \\
\hline Hp & 0.33 & 1.00 & 0.33 & 14 \\
\hline Lenovo & 0.20 & 3.00 & 1.00 & 0.24 \\
\hline
\end{tabular}


f. Alternative weight calculations for Reliability criteria

\section{a. Pairwise comparison}

\begin{tabular}{|c|c|c|c|}
\hline Reability & Samsung & $\mathrm{Hp}$ & Lenovo \\
\hline Samsung & 1 & 3 & $1 / 5$ \\
\hline $\mathrm{Hp}$ & $1 / 3$ & 1 & $1 / 3$ \\
\hline Lenovo & 5 & 3 & 1 \\
\hline
\end{tabular}

b. Criteria weight (vector priority), Being a decimal and sum up each column

\begin{tabular}{|c|c|c|c|}
\hline Reability & Samsung & Нp & Lenovo \\
\hline $\begin{array}{l}\text { Samsun } \\
\mathrm{g}\end{array}$ & 1 & 3 & 0.2 \\
\hline Hp & 0.33 & 1 & 0.33 \\
\hline Lenovo & 5 & 3 & 1 \\
\hline $\begin{array}{l}\text { Collumn } \\
\text { s }\end{array}$ & 6.33 & 7 & 1.53 \\
\hline
\end{tabular}

\begin{tabular}{|c|c|c|c|}
\hline \multicolumn{4}{|c|}{ Normalization } \\
\hline Style & Samsung & $\mathrm{Hp}$ & Lenovo \\
\hline Samsung & 0.16 & 0.43 & 0.13 \\
\hline $\mathrm{Hp}$ & 0.05 & 0.14 & 0.22 \\
\hline Lenovo & 0.79 & 0.43 & 0.65 \\
\hline
\end{tabular}

\begin{tabular}{|l|l|l|l|l|}
\hline \multicolumn{5}{|l|}{ Get the priority vector } \\
\hline Style & Samsung & HP & Lenovo & $\begin{array}{l}\text { Priority } \\
\text { Vector }\end{array}$ \\
\hline Samsung & 0.16 & 0.43 & 0.13 & 0.24 \\
\hline HP & 0.05 & 0.14 & 0.22 & 0.14 \\
\hline Lenovo & 0.79 & 0.43 & 0.65 & 0.62 \\
\hline
\end{tabular}

g. Calculation of alternative weights for Price criteria a. Pairwise comparison

\begin{tabular}{|l|l|l|l|}
\hline Price & Samsung & \multicolumn{1}{|c|}{ Hp } & Lenovo \\
\hline Samsung & 1 & 3 & 2 \\
\hline Hp & $1 / 3$ & 1 & 3 \\
\hline Lenovo & $1 / 2$ & $1 / 3$ & 1 \\
\hline
\end{tabular}

b. Criteria weight (vector priority), Being a decimal and sum up each column

\begin{tabular}{|l|l|l|l|}
\hline Reability & Samsung & $\mathrm{Hp}$ & Lenovo \\
\hline Samsung & 1.00 & 3.00 & 2.00 \\
\hline Hp & 0.33 & 1.00 & 3.00 \\
\hline Lenovo & 5 & 0.33 & 1 \\
\hline
\end{tabular}

Normalization

\begin{tabular}{|l|l|l|l|}
\hline Price & Samsung & $\mathrm{Hp}$ & Lenovo \\
\hline Samsung & 0.55 & 0.69 & 0.33 \\
\hline Hp & 0.18 & 0.23 & 0.5 \\
\hline Lenovo & 0.27 & 0.08 & 0.17 \\
\hline Collumn & 1.83 & 4.33 & 6.00 \\
\hline
\end{tabular}

Get the priority vector

\begin{tabular}{|c|c|c|c|}
\hline Style & Samsung $\mathrm{Hp}$ & Lenovo & Priority vector \\
\hline
\end{tabular}

\begin{tabular}{|l|l|l|l|l|}
\hline Samsung & 0.55 & 0.69 & 0.33 & 0.53 \\
\hline Hp & 0.18 & 0.23 & 0.5 & 0.30 \\
\hline Lenovo & 0.27 & 0.08 & 0.17 & 0.17 \\
\hline
\end{tabular}

h. Alternative ranking (the sum of the multiplication of each alternative weight with the weight of the corresponding criteria)

\begin{tabular}{|c|c|c|c|c|c|}
\hline & Style & Reability & Price & \multirow{4}{*}{$\mathrm{x}$} & $\begin{array}{l}\text { Priority } \\
\text { vector } \\
\end{array}$ \\
\hline Samsung & 0.62 & 0.24 & 0.53 & & 5.00 \\
\hline $\mathrm{Hp}$ & 0.14 & 0.14 & 0.30 & & 0.33 \\
\hline Lenovo & 0.24 & 0.62 & 0.10 & & 1.00 \\
\hline
\end{tabular}

\begin{tabular}{|l|ll|l|l|}
\hline & Style & Reability & Price & $\begin{array}{l}\text { Priority } \\
\text { vector }\end{array}$ \\
\hline Samsung & 0.43 & 0.05 & 0.05 & 0.53 \\
& $(0.62 \times$ & $(0.24 \times$ & $(0.53 \times$ & $(0.43+$ \\
& $0.70)$ & $0.20)$ & $0.10)$ & $0.05+$ \\
& & & & $0.05)$ \\
\hline Hp & 0.10 & 0.03 & 0.03 & 0.16 \\
& $(0.14 \times$ & $(0.14 \times$ & $(0.30 \times$ & $(0.10$ \\
& $0.70)$ & $0.20)$ & $0.10)$ & +0.03 \\
& & & & $+0.03)$ \\
\hline Lenovo & 0.17 & 0.13 & 0.02 & 0.32 \\
& $(0.24 \times$ & $(0.62 \times$ & $(0.17 \times$ & $(0.17$ \\
& $0.70)$ & $0.20)$ & $0.10)$ & $+0.13+$ \\
& & & & $0.02)$ \\
\hline
\end{tabular}

\section{IMPLEMENTATION RESULTS}

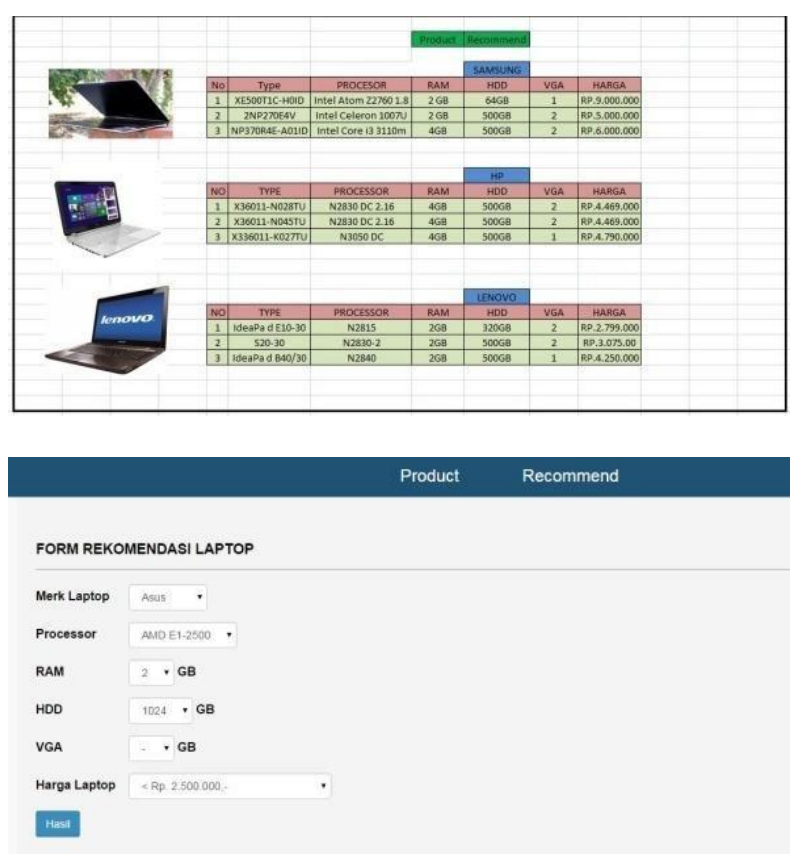

\section{CONCLUSIONS AND SUGGESTIONS}

\section{A. Conclusion}

Based on the response given by the client, it can be concluded that the decision support system that has been made is enough to help the client in choosing a laptop that suits his

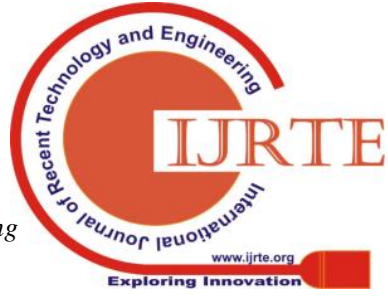




\section{Application of Analytical Hierarchy Process Method in Laptop Selection}

needs. Ranking results are in accordance with the above criteria

It obtained in the order namely: Samsung, HP and Lenovo, which have been inputted by clients such as laptop brands, processors, RAM, HDD, VGA, and prices.

\section{B. Suggestion}

1. In the future this DSS can be made online so that all users can use it as a reference for purchasing laptops.

2. Complete the brand and type of laptop and its specifications so that, the selection is not limited to a few brands.

3. Add images of each brand and type so that users can immediately see the picture of the laptop

\section{REFERENCES}

[1]. Elisabet Yunaeti Anggraeni, Pardimin, Ihsan Dacholfany, Akla, Miftachul Huda, Kamarul Shukri Mat Teh, Aminudin Hehsan, Juhazren Junaidi, Farahwahida Mohd Yusof, Hafiza Abas, Mohd Fauzi Abu Husin, Dina Apriani, Aliza Abdul Latif, Andino Maseleno, Modelling effectivenes of IS learning methodology with AHP method, International Journal of Engineering and Technology(UAE), Vol. 7, No. 4, 2018, pp. 4708-4714.

[2]. Andino Maseleno, Alicia Y.C. Tang, Moamin A. Mahmoud, Marini Othman, Suhendi Saputra, Muhamad Muslihudin, Fuzzy AHP Method to Determine Headache Types based on Symptoms, Investigacion Clinica, Vol. 58, No. 1, 2017.

[3]. K. Shankar, S.K. Lakshmanaprabu, Deepak Gupta, Andino Maseleno, Victor Hugo C. De Albuquerque, Optimal feature-based multi-kernel SVM approach for thyroid disease classification, The Journal of Supercomputing, Springer, Vol. 74, no. 259,2018 , pp. 1-16.

[4]. Noca Yolanda Sari, Miftachul Huda, Kamarul Shukri Mat Teh, Anggia Sari, Ramona Ramli, Andino Maseleno, Decision support system for determining chili plant using fuzzy multiple attribute decision making, International Journal of Engineering and Technology(UAE), Vol. 7, No. 4, pp. 3556-3562, 2018.

[5]. Satria Abadi, Miftachul Huda, Kamarul Shukri Mat Teh, Zulkiflee Haron, Mohd. Nasir Ripin, Aminudin Hehsan, Shamsul Sarip, Muhamad Rafiqi Hehsan, Musfika Amrullah, Andino Maseleno, Hazard Level of Vehicle Smoke by Fuzzy Multiple Attribute Decision Making with Simple Additive Weighting Method, International Journal of Pharmaceutical Research, Vol. 10, Issue 4, 2018.

[6]. Tri Susilowati, P. Manickam, G. Devika, K. Shankar, Latifah, Muhamad Muslihudin, Wahidah Hashim, Miftachul Huda, Aleksandr Aleeksevich Korostelev, Andino Maseleno, Decision Support System for Determining Lecturer Scholarships for Doctoral Study Using CBR (Case-Based Reasoning) Method, International Journal of Recent Technology and Engineering (IJRTE), 2019. pp. 3281-3290.

[7]. Muhamad Muslihudin, M. Ilayaraja, K. Sathesh Kumar, K. Shankar, Jauharotun Jamilah, Dita Novitasari, Miftachul Huda, Wahidah Hashim, Irina V. Rudenko, Andino Maseleno, Decision Support System in Kindergarten Selection using TOPSIS Method, International Journal of Recent Technology and Engineering (IJRTE), 2019. pp.3291-3298.

[8]. Muhamad Muslihudin, S. Hemalatha, K. Shankar, Eswaran Perumal, Nofiyanti, Satria Abadi, Wahidah Hashim, Andino Maseleno, Application of Expert System for Determining Export Quality Pepper Seeds using Website-Based Forward Chaining Method, International Journal of Recent Technology and Engineering (IJRTE), 2019. pp. 3319-3329.

[9]. Muhamad Muslihudin, Rizky Purnama, Wahidah Hashim, Andino Maseleno, Selection of Temporary Landfill using Fuzzy Multiple Attribute, International Journal of Innovative Technology and Exploring Engineering, 2019. pp.598-604.

[10]. Lakshmanaprabu, R.M. Vidhyavathi, Andino Maseleno, Charismatic Document Clustering through Novel K-Means Non-negative Matrix Factorization (KNMF) Algorithm using Key Phrase Extraction, International Journal of Parallel Programming,
Springer, 2018, pp. 1-19.

[11]. K. Shankar, S.K. Lakshmanaprabu, Deepak Gupta, Andino Maseleno, Victor Hugo C. De Albuquerque, Optimal feature-based multi-kernel SVM approach for thyroid disease classification, The Journal of Supercomputing, Springer, Vol. 74, no. 259,2018 , pp. 1-16.

[12]. M. Miftakul Amin, Adi Sutrisman, Deris Stiawan, Andino Maseleno, Design Restful WebService of National Population Database for supporting E-health interoperability service, Journal of Theoretical and Applied Information Technology, vol. 96, issue 15,2018

[13]. A.H. Motlagh, S.V. Klyuev, Aravindhan Surendar, Aygul Z. Ibatova, Andino Maseleno, Catalytic Gasification of Oil Sludge with Calcined Dolomite, Petroleum Science and Technology, Taylor and Francis, pp. 1-5, 2018.

[14]. Aravindhan Surendar, Alireza Bozorgian, Andino Maseleno, Lubov K. Ilyashenko, Meysam Najafi, Oxidation of Toxic Gases via Ge-B36N36 and Ge-C72 Nanocages as Potential Calaysts, Inorganic Chemistry Communications, Elsevier, Vol. 96, October 2018, pp. 206-210.

[15]. Abdolhamid Namdarian, Amin Goljanian Tabrizi, Andino Maseleno, Abdolkhaled Mohammadi, Seyyed Ebrahim Mossavifard, One step synthesis of rGO-Ni3S2 nano-cubes composite for high-performance supercapacitor electrodes, International Journal of Hydrogen Energy, Elsevier, vol. 43, Issue 37, 13 September 2018, pp.17780-17787.

[16]. Aravindhan Surendar, Lina G. Akhmetov, Lubov K. Ilyashenko, Andino Maseleno, Vahid Samavatian, Effect of thermal cycle loadings on mechanical properties and thermal conductivity of a porous lead-free solder joint, IEEE Transactions on Components, Packaging, and Manufacturing Technology, 2018, pp. 1769-1776.

[17]. Aravindhan Surendar, Vahid Samavatian, Andino Maseleno, Aygul Z. Ibatova, Majid Samavatian, Effect of solder layer thickness of thermo-mechanical reliability of a power electronic system, Journal of Material Science: Materials in Electronics, Springer, September 2018, Volume 29, Issue 17, pp. 15249-15258.

[18]. Majid Samavatian, Lubov K. Ilyashenko, Aravindhan Surendar, Andino Maseleno, Vahid Samavatian, Effect of System Design on Fatigue Life of Solder Joints in BGA Packages Under Vibration at Random Frequencies, Journal of Electronic Materials, November 2018, Volume 47, Issue 11, pp. 6781-6790.

[19]. Ilgar Javanshir, Andino Maseleno, Shahin Tasoujian, Majid Oveisi, Optimization of suspension system of heavy off-road vehicle for stability enhancement using integrated anti-roll bar and coiling spring mechanism, Journal of Central South University, September 2018, Volume 25, Issue 9, pp 2289-2298.

[20]. Andino Maseleno, Alicia Y.C. Tang, Moamin A. Mahmoud, Marini Othman, K. Shankar, Big Data and E-Learning in Education, International Journal of Computer Science and Network Security, 2018, Vol. 18, No. 5, pp. 171- 174.

[21]. Muhammad Assahubulkahfi, Yahaya Md. Sam, Andino Maseleno, Miftachul Huda, LQR Tuning by Particle Swarm Optimization of Full Car Suspension System, International Journal of Engineering and Technology(UAE), Vol. 7, No. 2.13, 2018, pp. 328-331.

[22]. Dwi Kurniasih Kamarul Azmi Jasmi, Bushrah Basiron, Miftachul Huda, Andino Maseleno, The uses of fuzzy logic method for finding agriculture and livestock value of potential village, International Journal of Engineering and Technology(UAE), Vol. 7, No. 3, 2018, pp. 1091-1095.

[23]. Hana Adela, Kamarul Azmi Jasmi, Bushrah Basiron, Miftachul Huda, Andino Maseleno, Selection of Dancer Member using Simple Additive Weighting, International Journal of Engineering and Technology(UAE), Vol. 7, No. 3, 2018, pp. 1096-1107.

[24]. Tri Susilowati, M. Ihsan Dacholfany, Sudirman Aminin, Afiful Ikhwan, Badlihisham Mohd. Nasir, Miftachul Huda, Adi Prasetyo, Andino Maseleno, Fiqih Satria, Sri Hartati, Wulandari, Getting Parents Involved in Child's School: Using Attendance Application System based on SMS Gateway, International Journal of Engineering and Technology(UAE), Vol. 7, No. 2.27, 2018, pp. 167-174.

[25]. Andino Maseleno, Alicia Y.C. Tang, Moamin A. Mahmoud, Marini Othman, Suhendi 
Saputra, Muhamad Muslihudin, Fuzzy AHP Method to Determine Headache Types based on Symptoms, Investigacion Clinica, Vol. 58, No. 1, 2017.

[26]. Andino Maseleno, Miftachul Huda, Maragustam Siregar, Roslee Ahmad, Aminudin Hehsan, Zulkiflee Haroon, Mohd Nasir Ripin, Siti Suhaila Ikhwani, Kamarul Azmi Jasmi, Combining the Previous Measure of Evidence to Educational Entrance Examination, Journal of Artificial Intelligence, Vol. 10, No. 3, pp. 85-90, 2017. ISSN: 2077-2173. doi: 3923/jai.2017.85.90

[27]. Miftachul Huda, Andino Maseleno, Kamarul Azmi Jasmi, Ismail Mustari, Bushrah Basiron, Strengthening Interaction from Direct to Virtual Basis: Insights from Ethical and Professional Empowerment, International Journal of Applied Engineering Research, Vol. 12, No. 17, pp. 6901-6909, 2017. ISSN: 0973-4562.

[28]. Miftachul Huda, Andino Maseleno, Masitah Shahrill, Kamarul Azmi Jasmi, Ismail Mustari, Bushrah Basiron, Exploring Adaptive Teaching Competencies in Big Data Era, International Journal of Emerging Technologies in Learning, Vol. 12, No. 3, pp. 68-83, 2017. ISSN: 1863-0383. doi: 10.3991/ijet.v12i03.6434.

[29]. P. Uma Maheswari, P. Manickam, K. Sathesh Kumar, Andino Maseleno, K. Shankar, Bat optimization algorithm with fuzzy based PIT sharing (BF-PIT) algorithm for Named Data Networking (NDN), Journal of Intelligent \& Fuzzy Systems, IOS Press, pp. 1-8, 2019.

[30]. Mohamed Elhoseny, K. Shankar, S.K. Lakshmanaprabu, Andino Maseleno, N. Arunkumar, Hybrid Optimization with Cryptography Encryption for Medical Image Security in Internet of Things, Neural Computing and Applications, Springer, October 2018, pp. 1-15.

[31]. E. Laxmi Lydia, P. Krishna Kumar, K. Shankar, S.K. Lakshmanaprabu, R.M. Vidhyavathi, Andino Maseleno, Charismatic Document Clustering through Novel K-Means Non-negative Matrix Factorization (KNMF) Algorithm using Key Phrase Extraction, International Journal of Parallel Programming, Springer, 2018, pp. 1-19.

[32]. K. Shankar, S.K. Lakshmanaprabu, Deepak Gupta, Andino Maseleno, Victor Hugo C. De Albuquerque, Optimal feature-based multi-kernel SVM approach for thyroid disease classification, The Journal of Supercomputing, Springer, Vol. 74, no. 259, 2018, pp. 1-16.

[33]. Satria Abadi, Akmal Hawi, Akla, Ihsan Dacholfany, Miftachul Huda, Kamarul Shukri Mat Teh, Jaki Walidi, Wahidah Hashim, Andino Maseleno, Identification of Sundep, Leahopper and Fungus of Paddy by using Fuzzy SAW Method, International Journal of Pharmaceutical Research, Volume 11, Issue 1, January - March 2019.

[34]. Elena V. Smirnova, B. Ayshwarya, Phong Thanh Nguyen, Wahidah Hashim, Andino Maseleno, Using Shell Programs in Educational and Methodological Support for Learning Foreign Language, International Journal of Applied Exercise Physiology, Vol. 8 (2.1), 2019. pp. 1027-1032.

[35]. Inga E. Rakhimbaeva, Aleksandr A. Korostelev, Indira A. Shakirova, B. Ayshwarya, Phong Thanh Nguyen, Wahidah Hashim, Andino Maseleno, Integration of the Educational and Didactic Systems in the Training of Future Teachers, International Journal of Applied Exercise Physiology, Vol. 8 (2.1), 2019. pp. 1033-1038.

[36]. Alla L. Busygina, Elena M. Chertakova, Darya B. Shtrikova, B. Ayshwarya, Phong Thanh Nguyen, Wahidah Hashim, Andino Maseleno, Professional forms of Employment in the Russian Federation: Problems and Challenges, International Journal of Applied Exercise Physiology, Vol. 8 (2.1), 2019. pp. 1039-1045.

[37]. Olga I. Pugach, Andrei V. Ochepovsky, Wahidah Hashim, Andino Maseleno, B.Ayshwarya, Phong Thanh Nguyen, To the use of English Words When Learning Programming, Information Systems and Technologies, International Journal of Applied Exercise Physiology, Vol. 8 (2.1), 2019. pp. 1046-1051.

[38]. Widodo, S., Miftakhul Amin, M., Bahri Joni M, A. 2019. Implementasion of Calibration in Gas Hazardous Carbon Monoxide, Carbon Dioxide and Methana in Closed Room Using Fuzzy Method, Journal of Physics: Conference Series, Volume 1167 , conference 1 , pp. 1-10 